\title{
A place for play? The influence of the home physical environment on children's physical activity and sedentary behaviour
}

\author{
Clover Maitland ${ }^{1 *}$, Gareth Stratton ${ }^{1,2}$, Sarah Foster ${ }^{3}$, Rebecca Braham ${ }^{1}$ and Michael Rosenberg ${ }^{1}$
}

\begin{abstract}
The home environment is an important influence on the sedentary behaviour and physical activity of children, who have limited independent mobility and spend much of their time at home. This article reviews the current evidence regarding the influence of the home physical environment on the sedentary behaviour and physical activity of children aged 8-14 years. A literature search of peer reviewed articles published between 2005 and 2011 resulted in 38 observational studies (21 with activity outcomes, 23 with sedentary outcomes) and 11 experimental studies included in the review. The most commonly investigated behavioural outcomes were television watching and moderate to vigorous physical activity. Media equipment in the home and to a lesser extent the bedroom were positively associated with children's sedentary behaviour. Physical activity equipment and the house and yard were not associated with physical activity, although environmental measures were exclusively self-reported. On the other hand, physical activity equipment was inversely associated with sedentary behaviours in half of studies. Observational studies that investigated the influence of the physical and social environment within the home space, found that the social environment, particularly the role of parents, was important. Experimental studies that changed the home physical environment by introducing a television limiting device successfully decreased television viewing, whereas the influence of introducing an active video game on activity outcomes was inconsistent. Results highlight that the home environment is an important influence on children's sedentary behaviour and physical activity, about which much is still unknown. While changing or controlling the home physical environment shows promise for reducing screen based sedentary behaviour, further interventions are needed to understand the broader impact of these changes. Future studies should prioritise investigating the influence of the home physical environment, and its interaction with the social environment, on objectively measured sedentary time and home context specific behaviours, ideally including technologies that allow objective measures of the home space.
\end{abstract}

Keywords: Home environment, Physical activity, Sedentary behaviour, Children, Adolescents, Review

\section{Introduction}

Changes to the environment in recent years have contributed to an increase in sedentary behaviour and a decline in activity [1]. The home environment is an important sphere of influence on the physical activity (PA) and sedentary behaviour of children. It is especially relevant for those who have limited independent mobility and spend

\footnotetext{
*Correspondence: clover.maitland@uwa.edu.au

'School of Sport Science, Exercise and Health, University of Western Australia, 35 Stirling Highway, Crawley, WA 6009, Australia

Full list of author information is available at the end of the article
}

much of their time at home and indoors [2,3], thereby potentially affecting PA participation and resultant health outcomes [4-6]. More recently, time spent sedentary, in particular watching television, has been associated with detrimental health effects including overweight and obesity, reduced fitness and poorer social and cognitive skills $[7,8]$. Still, many children do not meet health recommendations for PA and sedentary behaviour $[9,10]$. Thus, understanding the potential impact of the home environment on the sedentary and activity behaviours of children is vital for developing effective interventions.

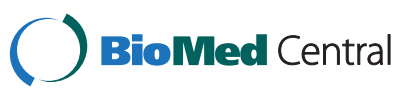


Sedentary behaviour and PA are part of a movement continuum [11]. PA can be of light, moderate or vigorous intensity and at home may include unstructured play, exercise and chores [12]. Sedentary behaviours use low levels of energy ( $\leq 1.5 \mathrm{METs}$ ) while sitting or reclining, such as watching television, using a computer and reading, and are distinct from insufficient PA, also termed as inactivity [1]. Notably, reviews have concluded the independence of moderate to vigorous physical activity (MVPA) and sedentary behaviour in children [13-16]. Therefore while sedentary behaviour and PA coexist in the home space, they are distinct behaviours influenced by different factors [11].

Ecological models emphasise individual, social and physical environmental influences on PA and sedentary behaviour [13,17-19]. Consequently, a large body of literature exists on social environmental influences [20] and the built environment at the neighbourhood level [21,22], while the home physical environment has received less attention. Qualitative studies have identified lack of yard space and sedentary entertainment options, such as televisions and computers, as barriers to children's PA, especially active play [23-26]. These factors, along with home design, have also been noted as influences on electronic media use [27]. However, previous reviews of correlates have not located any studies that have investigated the home physical environment with the exception of PA and media equipment $[13,14,16,28,29]$, or considered interactions between physical and social environmental influences within the home space.

Across the world, home environments are rapidly changing. House sizes in countries such as Australia and the USA have increased, while block and yard sizes have decreased [30]. In contrast lack of indoor and outdoor space is a concern in the UK [31,32]. Additionally, new electronic media technologies such as wireless broadband, multifunctional devices and interactive video games are now an integral part of homes in developed countries. Time use studies have found that leisure time at home indoors is more likely to be sedentary, while time at home in the garden is more likely to be active $[33,34]$. Accordingly, there is a potentially important link between location within the home space and children's PA and sedentary behaviours.

Thus, it is timely to review the influence of the home physical environment on children's PA and sedentary behaviour. The aims of this review were to: (1) examine the impact of interventions that change the home physical environment on children's PA and sedentary behaviours; (2) summarise the association between home physical environmental factors and children's PA and sedentary behaviours; (3) explore the relationship of physical and social environmental factors operating within the home space; and (4) highlight current evidence limitations, measurement issues and future research directions. The time of transition from childhood to adolescence, known as preadolescence, represents a specific stage [35] and has been chosen as PA levels decrease $[36,37]$ and sedentary screen based behaviour is high $[13,38]$. The review commences in 2005 to reflect recent changes in homes and build upon evidence from previous reviews of PA and sedentary behaviour by van der Horst et al. [14] and others [28,29].

\section{Methods}

\section{Search procedure}

Medline, Web of Science, PsychInfo and Sportdiscus databases were searched for quantitative studies examining the relationship between the physical home environment and preadolescent children's sedentary behaviours and PA. Combinations of key words were entered in three levels: children; activity; and home environment (Figure 1). The search was limited to English language peer reviewed journal articles published between 2005 and 2011.

\section{Inclusion/Exclusion criteria}

Inclusion criteria were: (1) sample of healthy children with mean age of between eight and 14 years (at baseline); and (2) outcome variable of sedentary behaviour or PA, including overall time spent sedentary or in PA, or time spent in specific behaviours that potentially occur within the home, such as television watching, video game play, active play and leisure time PA. While preadolescence has most commonly been defined as nine to 13 years [35], some studies have also included those aged eight and 14 years within this group [39]. Therefore, this age range was chosen to ensure all appropriate studies were included. Where studies included multiple age groups only the results from groups with a mean age of eight to 14 years were included in the review. Studies with outcomes of vigorous PA only, school based PA, active transport and structured sport were excluded. Observational studies were required to: (1) include at least one home physical environmental factor as an independent variable, for example home equipment or yard size; and (2) examine an association between the independent and outcome variable. Studies that included only neighbourhood level built environmental variables or home social environmental variables were excluded. Studies with combined independent measures, such as home and neighbourhood facilities, were also excluded. Experimental studies were included if they: (1) contained at least one strategy that changed the home physical environment, such as adding or removing equipment; and (2) reported changes from baseline in the outcome variable. 


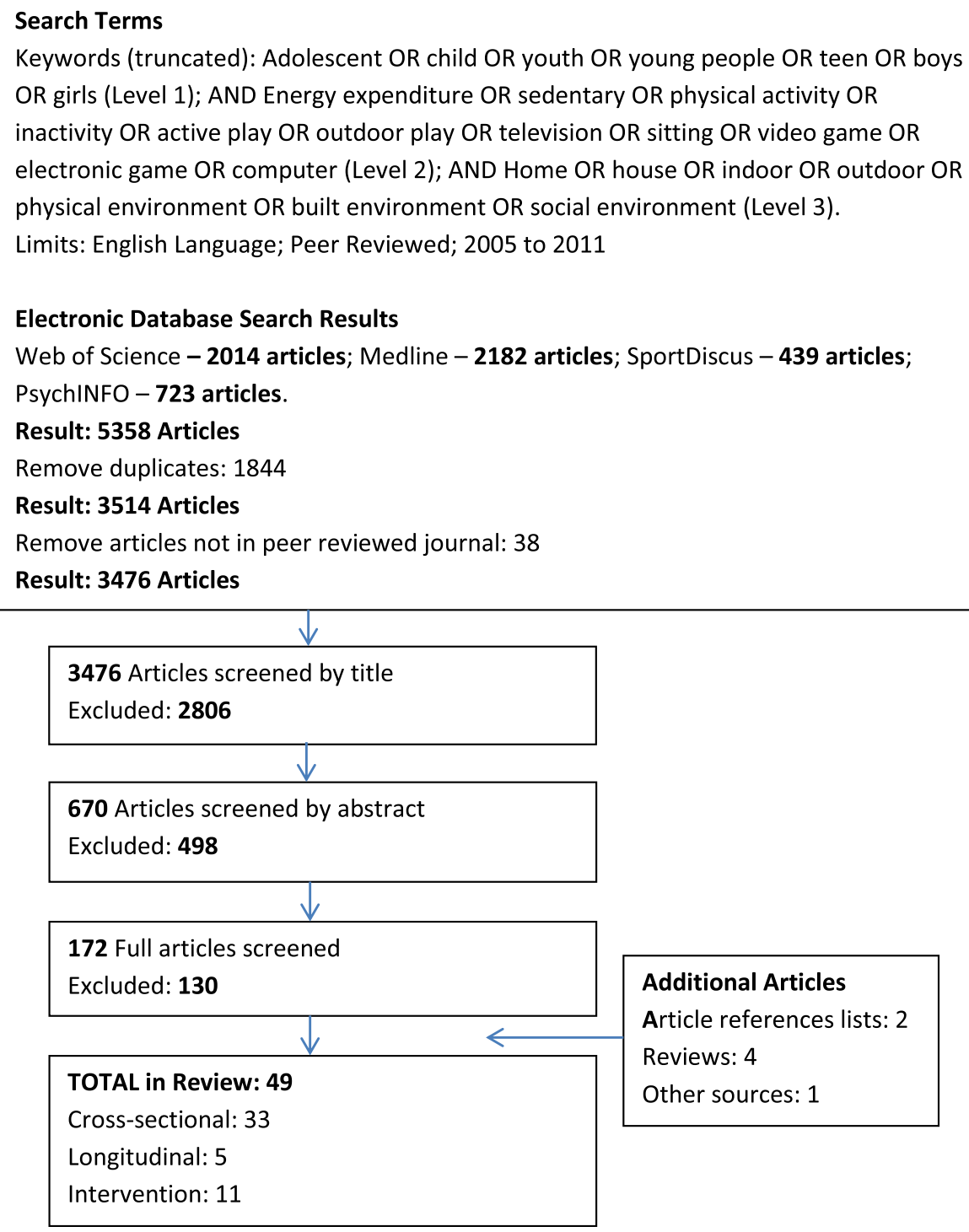

\begin{tabular}{|l|}
\hline \multicolumn{1}{|c|}{} \\
172 Full articles screened \\
Excluded: 130 \\
\hline $\begin{array}{l}\text { TOTAL in Review: } 49 \\
\text { Cross-sectional: } 33 \\
\text { Longitudinal: } 5 \\
\text { Intervention: } 11\end{array}$ \\
\hline
\end{tabular}

\section{Additional Articles}

Article references lists: 2

Reviews: 4

Other sources: 1

Figure 1 Literature search flow chart.

\section{Selection process}

Articles were screened in three phases. One reviewer read the title, then abstract and finally the full text, eliminating articles that did not meet the inclusion criteria at each screening phase. Twenty per cent of articles remaining at abstract level were independently screened by a second reviewer to confirm eligibility. Where ambiguity remained, a conclusion was reached by discussion between reviewers. Reference lists from selected articles and relevant review papers were searched for additional articles meeting inclusion criteria.

\section{Quality assessment}

To identify the best available evidence and provide a guide to quality, each paper was assessed according to
National Institute for Health and Clinical Excellence (NICE) quality appraisal checklists [40]. Each article received an overall score for internal and external validity, with '-' representing the lowest validity (few criteria fulfilled); '+' representing moderate validity (some criteria fulfilled); and ' ++ ' representing the highest validity (all or most criteria fulfilled).

\section{Data analysis}

Evidence tables summarising the study population, independent and outcome variables, analysis, results, quality assessment and intervention (where applicable), were constructed separately for observational and intervention studies according to NICE methods [40]. To summarise observational studies, all home physical environmental 
variables were categorised into: house and yard, PA equipment, media equipment, and bedroom media equipment. Home social environmental variables were categorised into: family rules, family social support (encouragement and co-participation/viewing) and family behaviour (sedentary behaviour and PA). As papers included a wide variety of sedentary behaviour and PA outcome variables, studies were designated as having either a sedentary or PA outcome and results were analysed in these two groups. Studies investigating both outcomes were included in both groups. Positive (+), negative (-) and null (0) associations significant at $\mathrm{p}<0.05$ from the highest level of multivariate analysis were extracted and are presented in Tables 1, 2 and 3, unless noted. Where studies analysed data by gender these results are reported separately in the Tables. For studies including analysis of multiple groups based on other criteria (e.g. age or country groups), time specific outcomes (e.g. weekend vs weekday screen time) or reporting methods (e.g. parent and self-report), at least half of the analyses must have shown an association in the reported direction. Results of studies were synthesised by totalling the number of studies reporting an association in a given direction. These totals are reported in the written results to provide overall trends for each of the key home environmental variables.

\section{Results}

Thirty-eight observational studies and 11 experimental studies were included in the final review (Figure 1). Most studies were conducted in high income countries including the USA $[49,51,52,55,59,61,72,74,75,77,79-83]$, Australia [43-45,54,67,69,71,73,76], UK [64-66,84], New Zealand [63,85-87] and The Netherlands [47,56,58,88]. Other countries contributing studies included China $[41,62]$, Spain [42,46], Canada [57,89], Italy [50], Belgium [60], Vietnam [68], Hong Kong [70] and France [78]. Two studies included multiple European countries [48,53].

\section{Observational studies}

Thirty-eight observational studies were identified including 33 cross-sectional and 5 longitudinal studies. Due to the low number of longitudinal studies, observational studies were analysed as one group. However, where applicable, the results of longitudinal studies are also reported separately to differentiate this stronger class of evidence. Nine studies scored the highest internal validity $[53,56,64,68,70-72,76,78]$ and twelve scored the highest external validity $[44,48,50,53,56,62,64,68,70-72,76]$.

Studies investigated a variety of outcomes with 17 measuring sedentary outcomes (Table 1), 15 measuring PA outcomes (Table 2), and six measuring both (Table 3). In studies with sedentary outcomes, the most common was TV time (15 studies), followed by sedentary time (6 studies), electronic game use (5 studies), screen time
(4 studies), computer/internet use (3 studies), mobile phone use ( 1 study) and reading ( 1 study). Two studies used accelerometers only [53,73] and one study used accelerometers with self-report to capture sedentary outcomes [77]. The remainder of studies used either self-report (13 studies [42-44,46-50,52,55-57,78]), parent report (3 studies $[45,54,76]$ ) or both (4 studies $[41,51,74,75])$.

PA outcomes included MVPA (11 studies), outdoor/ free play (4 studies), average activity level (3 studies), inactivity (3 studies), moderate PA (1 study) and light PA (1 study). Ten studies used accelerometers $[60,63,64,66$, 71-74,76,77], nine used self-report [59,61-63,65,68,70,75,78] and four used parental report $[58,67,69,75]$. Two incorporated more than one data collection method $[63,75]$.

\section{The home physical environment}

Measures of the home physical environment included media equipment in the home (25 studies), media equipment in the child's bedroom (20 studies), PA equipment (14 studies) and the parameters of the house and yard (6 studies). Two studies employed a home environment summary including both PA and media equipment [59,77]. Home physical environmental measures were collected via self-report in 19 studies [42-44,47,48,50,52,53,56,57,59,60, $63-66,70,73,78]$, parental report in 14 studies $[45,49,54,55,58$, 61,62,67-69,71,72,76,77] and five studies included both $[41,46,51,74,75]$. One study used an inventory implemented by parents [77] and no studies used objective measures of the home physical environment. Five studies reported the validity and reliability of the home physical environmental measure, ten reported reliability only and two reported validity only.

Media equipment within the home was positively associated with children's sedentary behaviours in 10 of 16 studies $[41,42,44-46,50,54,74,75,77]$. The one longitudinal study that investigated this relationship found no association between computers in the home and e-game or internet use [57]. For bedroom media equipment, nine of 18 studies found a positive association with sedentary behaviours $[41,43,45,50,51,54,56,75,78]$, including both longitudinal studies that investigated TV time [56,78]. One of these longitudinal studies also found an inverse relationship between a bedroom TV and reading [78]. There were limited and inconsistent associations between media equipment in the home and PA outcomes. Three of 14 studies found negative associations and two of these also found positive associations for different equipment measures $[66,70,76]$. Two of seven studies of bedroom media equipment found a positive association with PA $[58,73]$. The two longitudinal studies in this group showed no association between media equipment and PA $[71,78]$.

PA equipment was positively associated with PA outcomes in four of eleven studies $[61,63,75,77]$, with no 
Table 1 Summary of observational studies with sedentary behaviour outcomes only

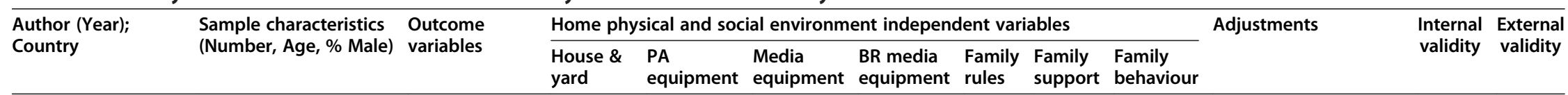

Sedentary Outcome Only - Cross-Sectional Studies Screen time
(SR,PR)

$+\quad+\quad 0 \quad+$

Age, income, sex, residence, clustering Not noted

Devis-Devis (2009); $\quad \mathrm{n}=323 ; 12-16$ yrs; $46 \%$
Spain $^{\mathrm{E}}$ [42]

TV time;

e-game time;

Mobile ph time

(SR) $(V, R)$

Granich (2011); $\quad n=298 ; 11-12 \mathrm{yrs} ; 49 \% \quad$ Screen time

Aust. [43] $\quad$ (SR) (R)

\begin{tabular}{|c|c|c|}
\hline $\begin{array}{l}\text { Hardy (2006); } \\
\text { Aust. [44] }\end{array}$ & $n=343 ; 12-13$ yrs; $50 \%$ & TV time $(S R)(R)$ \\
\hline $\begin{array}{l}\text { Hesketh (2007); } \\
\text { Aust. A B D [45] }^{\text {A }}\end{array}$ & $\mathrm{n}=895 ;$ grade $5 \& 6 ; 46 \%$ & TV Time (PR) (R) \\
\hline \multirow{2}{*}{$\begin{array}{l}\text { Hoyos Cillero (2011); } \\
\text { Spain [46] }\end{array}$} & $\mathrm{n}=503 ; 10-13$ yrs; $~ 50 \%$ & TV time; \\
\hline & & $\begin{array}{l}\text { Screen time } \\
\text { (SR) (R) }\end{array}$ \\
\hline
\end{tabular}

0

$+$

$+$

Hume (2010);
The Netherlands [47]

Jago (2008); Europe $\quad n=2670$; grade $3 \&$ 9; 49\% TV time;

[48]

e-game time

(SR)

$\begin{array}{lll}\begin{array}{l}\text { Norman (2005); } \\ \text { USA [49] }\end{array} & \mathrm{n}=878 ; 11-15 \text { yrs; } 46 \% & \begin{array}{l}\text { Sedentary time } \\ \text { (SR) (R) }\end{array} \\ & & \\ \begin{array}{l}\text { Patriarca (2009); } \\ \text { Italy [50] }\end{array} & \mathrm{n}=987 ; 11-16 \mathrm{yrs} ; 50 \% & \text { TV Time; } \\ & & \begin{array}{l}\text { Computer } \\ \text { time; }\end{array} \\ & & \text { e-game time } \\ & & \text { (SR) }\end{array}$

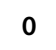

$\mathrm{OF}, \mathrm{OM}$

$-F, O M+F, O M$

$\begin{array}{llll}+ & 0 & 0 & + \\ + & + & - & + \\ +\mathrm{F}, \mathrm{OM} & \mathrm{OF}, \mathrm{OM} & \mathrm{OF},-\mathrm{M}\end{array}$

$\mathrm{OF}, \mathrm{OM}$

$\mathrm{OF}, \mathrm{OM}$

$\mathrm{OF}, \mathrm{OM}$

$\mathrm{OF},+\mathrm{M}$

$\mathrm{OF},+\mathrm{M}$

Not included (NS)

except for SES

(school day), gender

(weekend day); analysed

by school/weekend day

Not included (NS)

School clustering

Child \& parent BM

parent ed.; school

clustering; stratified

analysed by

week/weekend day

school clustering

Grade, gender,

father \& mother

income, obesity;

school clustering,

country (overall);

stratified by country

Age, ethnicity, BMI,

education; stratified

by gender

Age, gender, no. of

siblings, both parents

in household, parent

working activity,

sport activity 
Table 1 Summary of observational studies with sedentary behaviour outcomes only (Continued)

\begin{tabular}{|c|c|c|c|c|c|c|c|c|c|c|c|}
\hline \multirow{3}{*}{$\begin{array}{l}\text { Ramirez (2011); } \\
\text { USA [51] }\end{array}$} & \multirow[t]{3}{*}{ n=160; mean 14.6 yrs; 48\% } & \multirow{2}{*}{\multicolumn{3}{|c|}{$\begin{array}{l}\text { TV time; } \\
\text { e-game time; }\end{array}$}} & + & - & & & \multirow{3}{*}{$\begin{array}{l}\text { Not noted; analysed } \\
\text { by SR/PR }\end{array}$} & \multirow[t]{3}{*}{+} & \multirow[t]{3}{*}{+} \\
\hline & & & & & + & - & & & & & \\
\hline & & $\begin{array}{l}\text { Computer time } \\
(\mathrm{SR}, \mathrm{PR})(\mathrm{R})\end{array}$ & & & 0 & - & & & & & \\
\hline $\begin{array}{l}\text { Springer (2010); } \\
\text { USA [52] }\end{array}$ & $\mathrm{n}=734 ;$ grade $4 ; 50 \%$ & TV time $(S R)$ & & & 0 & - & & + & $\begin{array}{l}\text { Gender, ethnicity, } \\
\text { age, language, data } \\
\text { collection period, } \\
\text { school clustering; } \\
\text { analysed by } \\
\text { week/weekend day }\end{array}$ & + & + \\
\hline $\begin{array}{l}\text { van Sluijs (2010); } \\
\text { Europe [53] }\end{array}$ & $\begin{array}{l}n=2107 ; 9-10 \& 14-15 y r s ; \\
46 \& 56 \%\end{array}$ & $\begin{array}{l}\text { Sedentary time } \\
(\text { AcC })(V)\end{array}$ & & 0 & 0 & & & 0 & $\begin{array}{l}\text { Grade, sex; stratified } \\
\text { by country }\end{array}$ & ++ & ++ \\
\hline $\begin{array}{l}\text { van Zutphen (2007); } \\
\text { Aust. [54] }\end{array}$ & $n=1926 ; 4-12$ yrs; 49\% & TV time $(P R)$ & & + & + & - & & & $\begin{array}{l}\text { Parent ed., income, } \\
\text { SES, no. of adults } \\
\text { \& children }\end{array}$ & + & + \\
\hline $\begin{array}{l}\text { Zabinski (2007); } \\
\text { USA }^{C}[55]\end{array}$ & $\mathrm{n}=878 ; 11-15 \mathrm{yrs} ; 48 \%$ & $\begin{array}{l}\text { Sedentary time } \\
\text { (SR) }\end{array}$ & - & & & - & 0 & & Not noted & + & + \\
\hline \multicolumn{12}{|c|}{ Sedentary Outcome Only - Longitudinal Studies } \\
\hline $\begin{array}{l}\text { Te Velde (2011); } \\
\text { The Netherlands }{ }^{\mathrm{B}}[56]\end{array}$ & $\mathrm{n}=12654 ; 11-17 \mathrm{yrs} ; 54 \%$ & TV time (SR) (R) & & & + & - & & + & $\begin{array}{l}\text { Age, sex, school level } \\
\text { (SES), ethnicity }\end{array}$ & ++ & ++ \\
\hline \multirow{2}{*}{$\begin{array}{l}\text { Willoughby (2008); } \\
\text { Canada [57] }\end{array}$} & \multirow[t]{2}{*}{$n=1591 ;$ grade $9 \& 10 ; 51 \%$} & e-game use; & & 0 & & & & & \multirow{2}{*}{$\begin{array}{l}\text { Parent ed., baseline } \\
\text { value of outcome, gender }\end{array}$} & \multirow[t]{2}{*}{+} & \multirow[t]{2}{*}{+} \\
\hline & & $\begin{array}{l}\text { Internet use } \\
(\mathrm{SR})\end{array}$ & & 0 & & & & & & & \\
\hline
\end{tabular}

Longitudinal studies include age at baseline; E-games include electronic \& computer games; Computer includes computer and internet use; All analysis at highest multivariate level unless otherwise noted; Significance at $p<0.05$ unless otherwise noted; $M$ (male) \& F (female) entered separately where stratified as such; For studies with multiple groups or time specific outcomes at least half of analysis must show an association in the at $\mathrm{p}<0.05$ unless otherwise noted; $M$ (male) \& $\mathrm{F}$ (female) entered separately where stratified as such; For studies with multiple groups or time specific outcomes at least half of analysis must show an association in the
given direction ('t' or ' '-') with an independent variable; Adjustments listed as stated in paper; $S R$ self report, $P R$ parent report, Acc accelerometer, $V$ validity reported, $R$ reliability reported, $N S$ potential confounders not included in final analysis as found to be non-significant, SES socioeconomic status, $B M I$ body mass index, $A$ bivariate analysis, $B$ mediation analysis, $C$ cluster analysis, $D$ significance $p<0.1, E$ SEM analysis. 
Table 2 Summary of observational studies with physical activity outcomes only

\begin{tabular}{|c|c|c|c|c|c|c|c|c|c|c|c|c|}
\hline \multirow{2}{*}{$\begin{array}{l}\text { Author (Year); } \\
\text { Country }\end{array}$} & \multirow{2}{*}{$\begin{array}{l}\text { Sample characteristics } \\
\text { (Number, Age, \% Male) }\end{array}$} & \multirow{2}{*}{$\begin{array}{l}\text { Outcome } \\
\text { variables }\end{array}$} & \multicolumn{7}{|c|}{ Home physical and social environment independent variables } & \multirow[t]{2}{*}{ Adjustments } & \multirow{2}{*}{$\begin{array}{l}\text { Internal } \\
\text { validity }\end{array}$} & \multirow{2}{*}{$\begin{array}{l}\text { Externa } \\
\text { validity }\end{array}$} \\
\hline & & & $\begin{array}{l}\text { House \& } \\
\text { yard }\end{array}$ & $\begin{array}{l}\text { PA } \\
\text { equipment }\end{array}$ & $\begin{array}{l}\text { Media } \\
\text { equipment }\end{array}$ & $\begin{array}{l}\text { BR media } \\
\text { equipment }\end{array}$ & $\begin{array}{l}\text { Family } \\
\text { rules }\end{array}$ & $\begin{array}{l}\text { Family } \\
\text { support }\end{array}$ & $\begin{array}{l}\text { Family } \\
\text { behaviour }\end{array}$ & & & \\
\hline \multicolumn{13}{|c|}{ Physical Activity Outcome Only - Cross-Sectional Studies } \\
\hline $\begin{array}{l}\text { Aarts (2010); } \\
\text { The Netherlands [58] }\end{array}$ & $\begin{array}{l}n=4297 ; 7-9 y r s, 10-12 \text { yrs; } \\
\text { M/F }\end{array}$ & $\begin{array}{l}\text { Outdoor } \\
\text { Play (PR) }\end{array}$ & $+\mathrm{F},+\mathrm{M}$ & & $\mathrm{OF}, \mathrm{OM}$ & $+\mathrm{F},+\mathrm{M}$ & $\mathrm{OF}, \mathrm{OM}$ & & & $\begin{array}{l}\text { Parent ed., school clustering; } \\
\text { stratified by age, gender }\end{array}$ & + & + \\
\hline $\begin{array}{l}\text { Erwin (2007); } \\
\text { USA A B D }[59]\end{array}$ & $\mathrm{n}=47 ; 6-13 \mathrm{yrs} ; 70 \%$ & PA level (SR) & & 0 & 0 & & & & & None & - & - \\
\hline $\begin{array}{l}\text { Haerens (2009); } \\
\text { Belguim [60] }\end{array}$ & $\mathrm{n}=62 ;$ grade $7 \& 8 ; 19 \%$ & $\begin{array}{l}\text { MVPA time } \\
(A \subset C)(V, R)\end{array}$ & & 0 & 0 & & & 0 & & Age, SES, gender & + & + \\
\hline Kerr (2008); USA [61] & $\mathrm{n}=839 ; 11-15 \mathrm{yrs} ; 42 \%$ & $\begin{array}{l}\text { MVPA level } \\
(\mathrm{SR})(\mathrm{V})\end{array}$ & & $+\mathrm{F}, \mathrm{OM}$ & & & & & $\mathrm{OF}, \mathrm{OM}$ & $\begin{array}{l}\text { Age, ethnicity, parent ed., } \\
\text { parent PA; stratified by gender }\end{array}$ & + & - \\
\hline Li (2006); China [62] & $\mathrm{n}=1787 ; 11-17 \mathrm{yrs} ; 50 \%$ & $\begin{array}{l}\text { Inactivity } \\
\text { (SR) }\end{array}$ & $\mathrm{OF}, \mathrm{OM}$ & & $\mathrm{OF}, \mathrm{OM}$ & & $\mathrm{OF}, \mathrm{OM}$ & $\mathrm{OF},-\mathrm{M}$ & & Clustering; stratified by gender & + & ++ \\
\hline \multirow[t]{2}{*}{$\begin{array}{l}\text { Maddison (2009); } \\
\text { NZC }^{C} \text { [63] }\end{array}$} & $\mathrm{n}=110 ; 12-17 \mathrm{yrs} ; 57 \%$ & $\begin{array}{l}\text { MVPA time } \\
\text { (ACC); }\end{array}$ & & 0 & & & & & & Not noted & + & - \\
\hline & & $\begin{array}{l}\text { MVPA time } \\
(\mathrm{SR})(\mathrm{V})\end{array}$ & & + & & & & & & & & \\
\hline $\begin{array}{l}\text { McMinn (2011); } \\
\text { England [64] }\end{array}$ & $\mathrm{n}=2071 ; 9-10 \mathrm{yrs} ; 48 \%$ & $\begin{array}{l}\text { PA counts } \\
\text { (AcC) }\end{array}$ & 0 & & 0 & & & + & & $\begin{array}{l}\text { Sex, age quartiles, mth of } \\
\text { measurement, ethnicity, } \\
\text { school clustering }\end{array}$ & ++ & ++ \\
\hline Page (2010); UK [65] & $\mathrm{n}=1300 ; 10-11$ yrs; 50\% & $\begin{array}{l}\text { Outdoor } \\
\text { play (SR) }\end{array}$ & $\mathrm{OF}, \mathrm{OM}$ & & & & & & & $\begin{array}{l}\text { Daylight, neighbourhood } \\
\text { deprivation, pubertal stage, } \\
\text { BMl; stratified by gender }\end{array}$ & + & + \\
\hline $\begin{array}{l}\text { Ridgers (2010); } \\
\text { UK [66] }\end{array}$ & $n=110 ; 9-10 y r s ; 41 \%$ & $\begin{array}{l}\text { MVPA time } \\
(A \subset C)(V, R)\end{array}$ & & 0 & - & & & & & $\begin{array}{l}\text { School clustering; analysed } \\
\text { by week/weekend day time }\end{array}$ & + & + \\
\hline $\begin{array}{l}\text { Spinks (2006); } \\
\text { Aust. [67] }\end{array}$ & $\mathrm{n}=518 ; 5-12 \mathrm{yrs} ; 54 \%$ & $\begin{array}{l}\text { Inactivity } \\
\text { (PR) }\end{array}$ & & 0 & & & & & & $\begin{array}{l}\text { School clustering, gender, age, } \\
\text { maternal ed., school transport, } \\
\text { organised activity, family size, } \\
\text { TV/computer use }\end{array}$ & + & + \\
\hline $\begin{array}{l}\text { Trang (2009); } \\
\text { Vietnam [68] }\end{array}$ & $\mathrm{n}=2660 ; 11-16$ yrs; $50 \%$ & $\begin{array}{l}\text { Inactivity } \\
(S R)(V)\end{array}$ & OF,-M & & $\mathrm{OF}, \mathrm{OM}$ & & $\mathrm{OF}, \mathrm{OM}$ & $\mathrm{OF}, \mathrm{OM}$ & & $\begin{array}{l}\text { Clustering; sample weighted; } \\
\text { stratified by gender }\end{array}$ & ++ & ++ \\
\hline $\begin{array}{l}\text { Veitch (2010); } \\
\text { Aust. [69] }\end{array}$ & $\mathrm{n}=187 ; 8-9$ yrs; $53 \%$ & $\begin{array}{l}\text { Outdoor } \\
\text { play }(P R)(R)\end{array}$ & 0 & & & & & 0 & & $\begin{array}{l}\text { Not noted; analysed by } \\
\text { week/weekend day }\end{array}$ & + & + \\
\hline $\begin{array}{l}\text { Wong (2010); } \\
\text { Hong Kong [70] }\end{array}$ & $\begin{array}{l}\mathrm{n}=29,139 ; \text { mean } 14.5 / 6 \text { yrs; } \\
44 \%\end{array}$ & $\begin{array}{l}\text { MVPA time } \\
(S R)(V, R)\end{array}$ & & & $+/-\mathrm{F},-\mathrm{M}$ & & & & & $\begin{array}{l}\text { School clustering, age, family } \\
\text { affluence, parent ed.; stratified } \\
\text { by sex }\end{array}$ & ++ & ++ \\
\hline
\end{tabular}


Table 2 Summary of observational studies with physical activity outcomes only (Continued)

Physical Activity Outcome Only - Longitudinal Studies

Crawford (2010);

$\mathrm{n}=301 ; 10-12 \mathrm{yrs} ; 46 \%$

MVPA time

$\mathrm{OF}, \mathrm{OM}$

$\mathrm{OF}, \mathrm{OM}$

$\mathrm{OF}, \mathrm{OM}$

$+\mathrm{F}, \mathrm{OM}+\mathrm{F}, \mathrm{OM}$

$\mathrm{OF},+\mathrm{M}$

School clustering, baseline age,

average zBMl; analysed by sex

Wilson (2011)

$n=198$; grade $6 ; 47.5 \%$

MVPA time

0

Baseline MVPA \& BMl, free lunch,

ethnicity, parent ed., gender,

intervention, school clustering

Longitudinal studies include age at baseline; MVPA moderate to vigorous physical activity; Inactivity defined as insufficient MVPA; All analysis at highest multivariate level unless otherwise noted; Significance at $\mathrm{p}<0.05$ unless otherwise noted; $M$ (male) \& $\mathrm{F}$ (female) entered separately where stratified as such; For studies with multiple groups or time specific outcomes at least half of analysis must show an association in the given

direction (' + ' or ' '-) with an independent variable; Adjustments listed as stated in paper; $S R$ self report, $P R$ parent report, Acc accelerometer, $V$ validity reported, $R$ reliability reported, SES socioeconomic status, BMI body mass index, $A$ bivariate analysis, $B$ significance $p<0.01$; C SEM analysis; $D$ independent measure home environment summary including both physical activity and media equipment. 
Table 3 Summary of observational studies with sedentary behaviour and physical activity outcomes

\begin{tabular}{|c|c|c|c|c|c|c|c|c|c|c|c|c|}
\hline \multirow{2}{*}{$\begin{array}{l}\text { Author (Year), } \\
\text { Country }\end{array}$} & \multirow{2}{*}{$\begin{array}{l}\text { Sample characteristics } \\
\text { (Number, Age, \% Male) }\end{array}$} & \multirow{2}{*}{$\begin{array}{l}\text { Outcome } \\
\text { variables }\end{array}$} & \multicolumn{7}{|c|}{ Home physical and social environment independent variables } & \multirow[t]{2}{*}{ Adjustments } & \multirow{2}{*}{$\begin{array}{l}\text { Internal } \\
\text { validity }\end{array}$} & \multirow{2}{*}{$\begin{array}{l}\text { External } \\
\text { validity }\end{array}$} \\
\hline & & & $\begin{array}{l}\text { House } \\
\text { \& yard }\end{array}$ & $\begin{array}{l}\text { PA } \\
\text { equipment }\end{array}$ & $\begin{array}{l}\text { Media } \\
\text { equipment }\end{array}$ & $\begin{array}{l}\text { BR media } \\
\text { equipment }\end{array}$ & $\begin{array}{l}\text { Family } \\
\text { rules }\end{array}$ & $\begin{array}{l}\text { Family } \\
\text { support }\end{array}$ & $\begin{array}{l}\text { Family } \\
\text { behaviour }\end{array}$ & & & \\
\hline \multicolumn{13}{|c|}{ Sedentary and Physical Activity Outcome - Cross-Sectional Studies } \\
\hline \multirow[t]{3}{*}{$\begin{array}{l}\text { Hume (2005); } \\
\text { Aust. }{ }^{A B}[73]\end{array}$} & \multirow[t]{3}{*}{$\mathrm{n}=127 ; 10 \mathrm{yrs}, 52 \%$} & $\begin{array}{l}\text { Sedentary } \\
\text { time; }\end{array}$ & & $\mathrm{OF}, \mathrm{OM}$ & $\mathrm{OF}, \mathrm{OM}$ & $\mathrm{OF}, \mathrm{OM}$ & & & & \multirow[t]{3}{*}{ Not included (NS); stratified by sex } & \multirow[t]{3}{*}{+} & \multirow[t]{3}{*}{+} \\
\hline & & LPA; & & $\mathrm{OF}, \mathrm{OM}$ & $\mathrm{OF}, \mathrm{OM}$ & $\mathrm{OF}, 0 \mathrm{M}$ & & & & & & \\
\hline & & $\begin{array}{l}\text { MPA (AcC) } \\
(\mathrm{V}, \mathrm{R})\end{array}$ & & $\mathrm{OF}, \mathrm{OM}$ & $\mathrm{OF}, \mathrm{OM}$ & $\mathrm{OF},+\mathrm{M}$ & & & & & & \\
\hline \multirow[t]{3}{*}{$\begin{array}{l}\text { Roemmich (2007); } \\
\text { USA [74] }\end{array}$} & \multirow[t]{3}{*}{$\mathrm{n}=88 ; 8-12 \mathrm{yrs} ; 50 \%$} & $\begin{array}{l}\text { TV time } \\
(S R \& P R)\end{array}$ & & & + & & & & & \multirow[t]{3}{*}{$\begin{array}{l}\text { Age, SES, \% overweight, Acc wear } \\
\text { time (PA), gender }\end{array}$} & \multirow[t]{3}{*}{+} & \multirow[t]{3}{*}{+} \\
\hline & & PA counts; & & & 0 & & & & & & & \\
\hline & & $\begin{array}{l}\text { MVPA time } \\
(A c C)(V)\end{array}$ & & & 0 & & & & & & & \\
\hline \multirow{3}{*}{$\begin{array}{l}\text { Rosenberg (2010); } \\
\text { USA [75] }\end{array}$} & \multirow{3}{*}{$\begin{array}{l}\mathrm{n}=189 ; 12-18 \mathrm{yrs} ; 49 \% \\
\mathrm{n}=116 ; 5-11 \text { yrs; } 48 \%\end{array}$} & TV time; & & - & + & + & & & & \multirow{3}{*}{$\begin{array}{l}\text { Age, gender, race/ethnicity, household } \\
\text { income, no. of children; stratified by age; } \\
\text { analysed by PR/SR }\end{array}$} & \multirow[t]{3}{*}{+} & \multirow[t]{3}{*}{+} \\
\hline & & $\begin{array}{l}\text { Sedentary } \\
\text { time } \\
\text { (SR \& PR); }\end{array}$ & & 0 & + & + & & & & & & \\
\hline & & $\begin{array}{l}\text { MVPA time } \\
(S R \& P R)(V)\end{array}$ & & + & 0 & 0 & & & & & & \\
\hline \multirow[t]{2}{*}{$\begin{array}{l}\text { Salmon (2005); } \\
\text { Aust. [76] }\end{array}$} & \multirow[t]{2}{*}{$\mathrm{n}=878 ; 10-12 \mathrm{yrs} ; \mathrm{M} / \mathrm{F}$} & $\begin{array}{l}\text { TV Time } \\
(P R)(V, R) \text {; }\end{array}$ & & & $\mathrm{OF}, \mathrm{OM}$ & $\mathrm{OF}, 0 \mathrm{M}$ & $-F,-M$ & $\mathrm{OF},+\mathrm{M}$ & $+\mathrm{F}_{,}+\mathrm{M}$ & \multirow[t]{2}{*}{$\begin{array}{l}\text { Maternal ed., school clustering, } \\
\text { weight (girls TV model); stratified } \\
\text { by gender }\end{array}$} & \multirow[t]{2}{*}{++} & \multirow[t]{2}{*}{++} \\
\hline & & $\begin{array}{l}\text { PA counts } \\
\text { (low) } \\
\text { (AcC) }(V)\end{array}$ & & & $\mathrm{OF},+/-\mathrm{M}$ & $\mathrm{OF}, \mathrm{OM}$ & $-F,+M$ & $+\mathrm{F}, \mathrm{OM}$ & $+F_{,}+M$ & & & \\
\hline \multirow[t]{3}{*}{$\begin{array}{l}\text { Sirard (2010); } \\
\text { USA }{ }^{C}[77]\end{array}$} & \multirow[t]{3}{*}{$\mathrm{n}=575 ; 10-17 \mathrm{yrs} ; 49 \%$} & $\begin{array}{l}\text { Sedentary } \\
\text { time (AcC) } \\
(V, R) ;\end{array}$ & & - & + & 0 & & & & \multirow{3}{*}{$\begin{array}{l}\text { Gender, age, ethnicity, parent ed., } \\
\text { pubertal status, people in home, } \\
\text { parent BMl, mth of data collection, } \\
\text { free lunch, study cohort, school } \\
\text { clustering; stratified by gender } \\
\text { (screen time) }\end{array}$} & \multirow[t]{3}{*}{+} & \multirow[t]{3}{*}{+} \\
\hline & & $\begin{array}{l}\text { Screen } \\
\text { time }(S R)\end{array}$ & & $-F, 0 M$ & $+\mathrm{F}, \mathrm{OM}$ & $\mathrm{OF}, \mathrm{OM}$ & & & & & & \\
\hline & & $\begin{array}{l}\text { MVPA time } \\
(\text { AcC })(V)\end{array}$ & & + & 0 & 0 & & & & & & \\
\hline
\end{tabular}


Table 3 Summary of observational studies with sedentary behaviour and physical activity outcomes (Continued)

\section{Sedentary and Physical Activity Outcome - Longitudinal Studies}

\begin{tabular}{|c|c|c|c|c|c|}
\hline \multirow[t]{2}{*}{$\begin{array}{l}\text { Delmas (2007); } \\
\text { France [78] }\end{array}$} & \multirow[t]{2}{*}{$n=379,12 y r s ; 51 \%$} & $\begin{array}{l}\text { TV time; } \\
\text { Reading } \\
(S R)(V, R) ;\end{array}$ & \multirow[t]{2}{*}{$\begin{array}{l}\mathrm{OF},+\mathrm{M} \\
-\mathrm{F},-\mathrm{M} \\
\mathrm{OF}, \mathrm{OM}\end{array}$} & \multirow[t]{2}{*}{$\begin{array}{l}\text { School clustering, sexual maturity, } \\
\text { SES; stratified by gender }\end{array}$} & \multirow[t]{2}{*}{++} \\
\hline & & $\begin{array}{l}\text { Free \& } \\
\text { Club PA } \\
(S R)(V)\end{array}$ & & & \\
\hline
\end{tabular}

Longitudinal studies include age at baseline; PA physical activity, LPA light physical activity, MPA moderate physical activity, MVPA moderate to vigorous physical activity; All analysis at highest multivariate level unless otherwise noted; Significance at $\mathrm{p}<0.05$ unless otherwise noted; $M$ (male) \& $\mathrm{F}$ (female) entered separately where stratified as such; For studies with multiple groups or time specific outcomes at least half of analysis otherwise noted; Significance at $p<0.05$ unless otherwise noted; $M$ (male) \& $F$ (female) entered separately where stratified as such; For studies with multiple groups or time specific outcomes at least half of analys $\quad$ must show an association in the given direction (' $+t^{\prime}$ or ' - ) with an independent variable; Adjustments listed as stated in paper; $S R$ self report, $P R$ parent report, Acc accelerometer, $V$ validity reported, $R$ reliability

must show an association in the given direction ( $\left(+{ }^{\prime}\right.$ or ' - ') with an independent variable; Adjustments listed as stated in paper; $S R$ self report, $P R$ parent report, $A c c$ accelerometer, $V$ validity reported, $R$ reliability
reported, $N S$ potential confounders not included in final analysis as found to be non-significant, $S E S$ socioeconomic status, BMI body mass index, $A$ bivariate analysis; $B$ independent measures taken from map drawn by reported, NS potential confounders not included in final analysis as found to be non-significant
sample; C PA equipment measure also includes physical activity to media equipment ratio. 
association found in the two longitudinal studies [71,72]. On the other hand, PA equipment was inversely associated with sedentary behaviours in three of six studies $[55,75,77]$, although no longitudinal studies investigated this relationship. The least investigated category of the home physical environment was the house and yard. Two of six cross-sectional studies found that yard space was positively associated with a PA measure $[58,68]$ and one of these studies also found living in an apartment was negatively associated [58]. No studies investigating sedentary behaviours included a measure of size, space or design of the house and yard.

\section{Relationships between home physical and social environmental factors}

The majority of observational studies investigating the home physical environment also investigated home social environmental variables. Measures included family rules (19 studies), family social support (14 studies) and family behaviours (10 studies). Significant relationships between the home social environment and sedentary behaviours, and to a lesser extent PA, were evident after adjusting for home physical environmental factors. Some form of family social support was positively associated with sedentary behaviours in six of seven cross-sectional studies [41,43-45,49,76]. For PA outcomes five of eight studies showed an association in the expected direction. Social support for PA was positively associated with PA in four studies, including two longitudinal studies $[62,64,71,72]$, and playing e-games with parents was positively associated with low activity in one study [76]. Four of these studies scored the highest internal and external validity $[64,71,72,76]$. Six of eight studies on electronic media use, with a family behaviour measure, found parental electronic media use positively associated with children's use $[44,46,47,52,56,76]$. This included all studies investigating TV time. Of the three studies measuring parental behaviour and PA outcomes, one cross-sectional study found parent e-game use positively associated with low activity [76], and one longitudinal study found parent PA participation positively associated with MVPA [71]. Both studies scored the highest internal and external validity. Of the six studies investigating rules and PA, these same two studies found an association. The longitudinal study found a positive relationship between rules restricting PA and MVPA in girls [71], and the cross-sectional study found mixed results with supervision of TV positively associated with low activity in boys and negatively associated in girls [76]. Electronic media rules were negatively associated with sedentary behaviours in 11 of 14 studies, including the one relevant longitudinal study $[43,45,46,49-52,54-56,76]$.

In the 15 studies with sedentary outcomes that measured both physical and social environmental variables,
10 found associations with physical and social environmental measures [41,43-46,50,51,54-56]. Of these, only one study investigated an interaction between the home physical and social environmental factors reviewed, and results showed an inverse association between parental rules and TV viewing only when there was a TV in the bedroom [52]. In the 10 studies with PA outcomes that investigated the home physical and social environment, one study found associations with both [76]. No other moderating or mediating relationships between the reviewed physical and social environmental factors were explored.

\section{Experimental studies}

Experimental studies used one of two strategies to change the home physical environment: either introducing a television limiting device or an active video game (AVG).

\section{Television limiting device studies}

Five randomised control trials (RCT), ranging in duration from six weeks to 12 months, introduced a television limiting device (Table 4). Four studies scored the highest internal validity [79-81,89], while no studies scored the highest external validity. All studies measured screen based sedentary outcomes [79-81,85,89], and four also measured PA and body composition outcomes [79-81,89]. Three studies of the highest internal validity found a significant decrease in TV viewing in the intervention group (47, 73 and 116 minutes per day) $[80,81,89]$. Two of these also showed improvement in body mass index (BMI) $[81,89]$. The one study that rewarded children for PA with TV viewing tokens also increased PA by $65 \%$ [89]. Of the two studies that did not significantly change children's sedentary behaviours, one found a significant decrease in overall household TV watching [79].

\section{Active video gaming studies}

Of the six experimental studies that introduced an AVG into the home, four were RCTs (Table 5). Two RCTs scored the highest internal validity $[84,86]$, while no studies scored the highest external validity. Study durations ranged between 12 weeks and six months. Five of six studies collected outcome measures mid-intervention. Of the three RCTs that compared an intervention group to a play as usual control group: one found an increase in AVG play of 57 minutes per day at mid-intervention [84]; one found an increase in AVG play of 10 minutes per day and improvement in BMI post-intervention [86]; and one found an average difference in sedentary video gaming of 52 minutes per day, an increase in PA at mid-intervention and improvement in waist circumference post-intervention [87]; Two studies found a significant decrease in AVG play between the first and second half of the intervention $[83,84]$, with another two showing non-significant decreases [82,88]. 
Table 4 Summary of experimental studies including TV limiting devices

\begin{tabular}{lll}
\hline Author (Year); & Sample characteristics & Intervention description \\
Country & (Number; Age; Sex; Other) & (Design; Duration; Characteristics)
\end{tabular}

French (2011); $\quad \mathrm{n}=75$ adolescents randomised, Cluster RCT; 12 mths; Intervention -

USA [79] $\quad \mathrm{n}=87 \mathrm{HHs}$; 12-17 yrs; Sex not TV limiting devices, guidelines about reported; HH TV $\geq 10$ hrs per food availability, 6 × group sessions, person per wk.

SB -

SB - TV (SR); PA - MVPA (SR);
Summary of key findings

SB - TV (SR); PA - MVPA (SR); $\quad$ Significant decrease in reporting

Other - zBMI, dietary intake, TV is on, and significant increase eating behaviours, PA in consumption of fruit and veg in intervention compared to control. At HH level there was a significant decrease in TV watching, and a significant with others in $\mathrm{HH}$ compared to control.

Goldfield (2006); $\quad n=30 ; 8-12$ yrs; 43\% M; Canada [89]

RCT. 8 wks; Intervention - wore PA overweight or obese, TV/video monitor (open-loop feedback) and games $\geq 15$ hrs per wk, $<30$ mins MVPA per day. rewarded for PA (reinforcement) with TV access via token controlled TV TV access via token controlled TV
limiting device; Control - wore a PA monitor (open-loop feedback only).

Ni Mhurchu (2009); $\mathrm{n}=29 ; 9-12 \mathrm{yrs} ; 62 \% \mathrm{M}$; New Zealand [85] TV > 20 hrs per wk.

RCT (Pilot); 6 wks; Intervention electronic TV monitors, encouraged to restrict TV to 60 mins per day, ideas to reduce TV. Control - ideas to from snacks; Interviews. reduce TV.

SB - TV based, other (SR); PA Significant total activity counts and MVPA (Acc): Other - height, weight, and reduction in TV based SB, fat BMI, dietary intake. intake, calories from snacks and snack intake in front of TV, and improvement in weight and BMI, compared to control. Reductions in weight, fat intake, calories from snacks, calories consumed in front of TV significantly correlated with reduction in TV based SB.

SB - TV, total screen time (SR); No significant differences. Decrease in weekly TV of 254 mins in intervention and 3 mins in control (NS). Total screen time decreased and steps increased slightly in both groups (NS) Mixed views on family acceptability of TV time monitors. Adjustments Internal External validity validity

Gender, smoking, ++ +
age, $\mathrm{HH}$ income, configuration, race, education; baseline values of outcomes; $\mathrm{HH}$ clustering.

Not noted.

Significant reduction in weekday TV, and weekday and weekend video game play compared to control. Significant reduction in mother, father and sibling TV viewing compared to control. Age, supervision, and prior TV and video game use moderated intervention effects. SMART school curriculum (18 lessons
with TV Turn Off Challenge and goal to reduce to 7 hrs per wk), TV member TV viewing: allowance device, parent newsletters; Control - no intervention. 
Table 4 Summary of experimental studies including TV limiting devices (Continued)

\begin{tabular}{|c|c|c|c|c|c|c|}
\hline $\begin{array}{l}\text { Todd (2008); } \\
\text { USA [81] }\end{array}$ & $\begin{array}{l}\mathrm{n}=21 ; 8-11 \text { yrs; } \mathrm{M} \text { only; } \\
\mathrm{TV}>3.5 \text { hrs or } \mathrm{EM}>5.8 \mathrm{hrs} \\
\text { per day. }\end{array}$ & $\begin{array}{l}\text { RCT; } 20 \text { wks; Intervention - seminar } \\
\text { including goal setting, newsletters, TV } \\
\text { allowance device, software to limit } \\
\text { computer use, phone calls, } \\
\text { recommendation to reduce EM to } 90 \\
\text { mins per day; Control - no } \\
\text { intervention. }\end{array}$ & $\begin{array}{l}\text { SB - EM use (SR); PA - steps } \\
\text { (pedometer); Other - height, } \\
\text { weight, BMI, \% body fat, } \\
\text { snacks and meals consumed } \\
\text { with EM, dietary intake, bone } \\
\text { mineral density. }\end{array}$ & $\begin{array}{l}\text { Significant treatment by time } \\
\text { interaction for EM use and } \% \\
\text { body fat. Intervention decreased } \\
\text { EM use from } 153 \text { mins per day to } \\
81 \text { ( } 10 \text { wks) and } 82 \text { ( } 20 \text { wks) and } \\
\text { control from } 157 \text { to } 119 \text { and } 95 \\
\text { (adjusted difference of } 73 \text { mins at } \\
20 \text { weeks); Intervention decreased } \\
\% \text { body fat from } 26.1 \text { to } 24.6 \text { ( } 20 \\
\text { wks) and control increased from } \\
27.7 \text { to } 28.0 \text {. Significant reduction } \\
\text { in snacks and meals consumed } \\
\text { with EM, compared to control. }\end{array}$ & $\begin{array}{l}\text { Organised activity, } \\
\text { electronic media } \\
\text { access. }\end{array}$ & ++ \\
\hline
\end{tabular}

$M$ male, $H H$ household, $S B$ sedentary behaviour, PA physical activity, $R C T$ randomised control trial, $M V P A$ moderate to vigorous physical activity, NS non-significant; $\mathrm{n}=$ is number in analysis unless noted; $B M I$ body mass index, $E M$ electronic media, Acc accelerometer, $S R$ self reported; Measures column includes outcomes and variables used in further analysis (excluding adjustment variables); Significance at $p<0.05$ (outcomes not reported or listed in table as non-significant if $\mathrm{p}>0.05)$; Adjustments listed as stated in paper. 
Table 5 Summary of experimental studies including active video games

\begin{tabular}{lll}
\hline Author (Year); & $\begin{array}{l}\text { Sample characteristics } \\
\text { Country }\end{array}$ & $\begin{array}{l}\text { Intervention description } \\
\text { (Number; Age; Sex; Other) }\end{array}$ \\
(Design; Duration; Characteristics)
\end{tabular}

Chin A Paw (2008); $\quad \mathrm{n}=16$; 9-12 yrs; 14\% M; $\quad$ RCT (Pilot); 12 wks; Multiplayer

The Netherlands [88] low fitness.

Graves (2010); $\quad \mathrm{n}=42 ; 8-10 \mathrm{yrs}$; \% not $\quad \mathrm{RCT} ; 12$ wks; Intervention - video

England [84] reported.

games linked to jOG device that required stepping; Control - video game play as usual.

A - Step powered video gaming, AVG play, total video gaming (SR) steps, CPM, total PA (Acc); SB sedentary video gaming, $\mathrm{TV}$ productive behaviours, leisure behaviours (SR), sedentary (Acc): Other-stature, body mass, BMI, Other - stature, body mass, BMI, maturity offset, subtotal body fat, trunk body fat.

Maddison (2011): $\quad \mathrm{n}=322 ; 10-14 \mathrm{yrs} ; 73 \% \mathrm{M}$; New Zealand [86] overweight/ obese, video games $\geq 2$ hrs per wk.

RCT; 24 wks; Intervention - AVG play as usual.

- sedentary video gaming (SR); Other - weight, BMI, zBMl, to body fat, $\%$ body fat, waist circumference, energy intake from snacks, fitness.

Pre/post design; 6 mths;

Madsen (2007); $\quad \mathrm{n}=30^{\mathrm{A}} ; 9-18 \mathrm{yrs} ; 40 \% \mathrm{M}$ USA [82] obese.

Ni Mhurchu (2008); New Zealand [87]

PA - Dance Dance Revolution group. (memory card); Other - BM Interviews. PA - AVG time, total video gaming (SR), CPM (ACC), MVPA (SR); SB -

RCT (Pilot Study); 12 wks; Intervention - AVG supplied, intervention - Interactive Dance

Simulation Video Game (IDSVG) for

home use, 60 min weekly group

class; Home intervention - IDSVG for

Measures

Summary of key findings

Adjustments Internal External

validity validity

PA - ISDVG play (SR); Other - focus

Multiplayer group averaged 901

Not noted.

$+$

groups.

mins ISDVG play and home group

376 mins (NS); Median play

decreased from 228 mins in first 6

home use only.

for home group, and increased

from $475 \mathrm{~min}$ to $601 \mathrm{~min}$ in

multiplayer group (NS).

multiplayer group (NS).
Significantly lower drop out in

Significantly lower drop out in
multiplayer group (15\%) compared

to home group (64\%); Technical

difficulties, need for computer and

space, dull music and becoming

bored were barriers.

Significant increase in AVG play compared to control at 6 weeks; step powered video gaming was significantly higher at week 6 than 12 in intervention group.

outcomes;

maturity

offset (some).

Significant treatment effect on zBMI, BMI, \% body fat, total body fat, and increase in active video game time compared to control.

Age, sex, ethnicity: baseline values of outcomes.

No significant effects. 12 children used DDR at least twice a week in first 3 months, and only 2 in second 3 months. Family stressors and boredom were barriers.

Baseline zBMI. instructed to substitute for regular video games;
Average time in inactive video gaming was significantly lower Sex; baseline values of
(CPM) (6 wks) significantly higher and waist circumference (12 wks) significantly improved compared to control. Average total video game time was lower (54 vs 98 mins per day) compared to control (NS). 
Table 5 Summary of experimental studies including active video games (Continued)

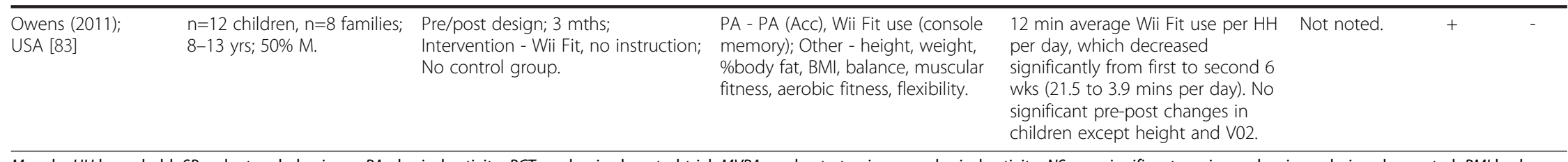

$M$ male, $H H$ household, $S B$ sedentary behaviours, $P A$ physical activity, $R C T$ randomised control trial, $M V P A$ moderate to vigorous physical activity, NS non-significant; $\mathrm{n}=$ is number in analysis unless noted; $B M I$ body mass index, Acc accelerometer, $S R$ self reported, CPM counts per minute; Measures column includes outcomes and variables used in further analysis (excluding adjustment variables); Significance at $p<0.05$ (outcomes not reported or listed in table as non-significant if $\mathrm{p}>0.05$ ); Adjustments listed as stated in paper; $A \mathrm{n}=26$ at $3 \mathrm{mths}, 21$ at $6 \mathrm{mths}, 12$ with DDR use diary, 7 had memory card. 


\section{Discussion}

The purpose of this paper was to review the influence of the home physical environment on children's PA and sedentary behaviour. Results showed that media equipment was positively associated with screen based sedentary behaviours and PA equipment was unrelated to PA, reinforcing results of earlier reviews. Several previously unreviewed relationships were summarised, highlighting an inverse relationship between PA equipment and sedentary behaviour in half of studies. Interventions that changed the home environment by introducing TV limiting devices reduced TV time. The social environment, in particular parents, played an important role in influencing children's sedentary behaviour and PA even in the presence of home physical environmental factors. The field is limited by the lack of objective assessment and no investigation of the indoor home space beyond equipment. Additionally, there was a paucity of studies investigating objectively measured sedentary time and home context specific behaviours. This review extends previous knowledge by critically assessing and synthesising evidence from both experimental and observational studies. The paper identifies current evidence gaps and measurement issues, and generates future directions for research on children's sedentary behaviour and PA within the home space.

\section{Research evidence and gaps \\ The home - house and yard}

In the current review, investigation of the size, space and design of the house and yard was limited. Although, previous reviews of correlates of children's physical activity have not addressed this relationship at all $[14,15,28,29]$. Only six studies could be found that collected any measure of the house or yard and the presence of a garden was the only measure collected more than once. While one of three studies investigating outdoor play found that girls without a garden played outside less [58], our overall results are in contrast to the findings of several qualitative studies that have identified lack of yard space as a barrier to physical activity and active play $[23,24,26]$. This lack of association could have been influenced by the limited and categorical nature of environmental variables investigated, and outcome measures that were not specific to the home. With the majority of children's MVPA occurring outside of the home [90,91], and the majority of leisure time at home spent indoors and sedentary [33,34], it would seem pertinent to investigate the influence of the house and yard on children's sedentary behaviour independent of PA. Further investigation of the indoor home space may also be relevant for PA with a recent ecological momentary assessment study of 9-13 year olds in California finding that $30 \%$ of all leisure time PA occurred indoors at home and only $8 \%$ occurred in the yard at home [90]. To date there has been no exploration of the relationship between the house and yard and children's sedentary behaviours or home context specific PA.

\section{The home - media equipment}

Observational studies showed a positive relationship between media equipment and children's screen based sedentary behaviours. This extends the findings of earlier reviews that have located few or no studies investigating this relationship $[13,14]$ and concurs with a more recent review of sedentary behaviour correlates [16]. The review also considered media equipment and PA, a relationship not summarised by previous reviews [14,15,28,29], and found the majority of studies showed no relationship.

This review located three studies investigating media equipment and objectively measured sedentary time and, to our knowledge, is the first review to summarise this relationship. Findings on the influence of media equipment in the home were mixed and there was no association between bedroom media equipment and accelerometer measured sedentary time [53,73,77]. Even with limited evidence, these findings are curious given the associations between media equipment and screen based sedentary behaviour. This suggests that children with less media equipment at home may simply substitute one sedentary behaviour for another resulting in no discernible difference to overall sedentary time. This is consistent with qualitative findings that indicated children would consider both active and sedentary alternatives if screen viewing was limited [25]. Notably, the studies reviewed measured sedentary time across the entire day. This included sedentary time in school and other places outside the home, which may be less likely to be influenced by home media equipment.

\section{The home - physical activity equipment}

Overall, our results support previous conclusions of limited evidence for a relationship between PA equipment at home and children's PA [14,28,29]. However, we also found that children with more PA equipment spent less time in sedentary behaviours in half of studies $[55,75,77]$, a relationship not considered by previous reviews $[13,14,16]$. While this evidence is inconclusive, it does raise the possibility that PA equipment at home may decrease sedentary behaviours by prompting alternative light intensity activities rather than MVPA.

\section{Changing the home environment}

Changing the home physical environment has the potential to influence children's sedentary behaviour and PA. This review found interventions that implemented a TV limiting device were successful in decreasing children's screen based sedentary behaviour. Also, introducing an AVG to the home resulted in positive changes in AVG 
play, sedentary electronic game play, PA and/or body composition in some studies $[84,86,87]$, although early changes did not always last. Interventions that showed the most promise in changing behaviour and/or body composition directed participants to substitute activities, such as active gaming for sedentary gaming, or to earn TV time by participating in PA $[84,87,89]$. However, the acceptability of these changes in the home was debatable, with the need for space, boredom and disruption of other family members cited $[82,85,88]$. Additionally, the long term effects of introducing an AVG or limiting TV viewing on other behaviours, including screen based sedentary behaviours, productive sedentary behaviours and $\mathrm{PA}$, are unclear.

\section{Home physical and social environmental interactions}

The review reaffirms the important role that parents play in supporting, restricting and normalising children's sedentary behaviours $[13,16]$. In accordance with ecological frameworks, we found that the influence of social environmental factors in the presence of physical environmental factors was evident. Although, it should be noted that results relating to the home social environment are limited to studies that investigated both social and physical environmental factors. Children with parents who watched more TV and those in families with no electronic media rules, spent more time watching TV. Additionally, children who participated in sedentary behaviour with their parents spent more time in sedentary behaviour. Despite many studies based upon ecological models, few investigated relationships between physical and social environmental factors within the home space. Of those that did, one study found parental rules were only effective when there was a bedroom TV [52]. Parents largely dictate the arrangement of the home space and determine the equipment available to children at home. Family rules are of particular interest as they present an avenue for controlling the influence of the physical environment through not allowing screens in bedrooms and living areas or choosing not to purchase media equipment. They may also restrict or encourage the use of home space for active behaviours. Thus far few studies have explored these relationships.

\section{Measurement issues}

Other authors have called for behaviour specific measures of the environment and context specific measures of PA and sedentary behaviours in the investigation of environmental influences $[19,92,93]$. Our review affirms the need for increased specificity of research on the home environment. There was only one study with a home context specific PA outcome [69]. Also, while specific sedentary behaviours such as TV watching mostly occur at home, objectively measured sedentary time was accumulated across the entire day. Reinforcing the value of this approach, we found elements of the home physical environment were more consistently associated with sedentary behaviours, which are more likely to occur at home, than with PA outcomes.

Studies of the neighbourhood built environment have used objective measures (audits and Geographic Information Systems (GIS)) as well as perceived measures (self-report) [22,94] to assess the environment, and Global Positioning Systems (GPS) to track individuals' movement [95]. This review found all but one study used surveys to measure the home environment, with the exception using an inventory that measured equipment density, availability and accessibility [77]. Supporting the case for more robust measurement, this study was the only one to find PA equipment, in this case equipment density, related to accelerometer measured MVPA. It also found the ratio of PA to media equipment was related to accelerometer measured sedentary time. While it is acknowledged that current GIS and GPS technologies provide limited utility indoors, other technologies combining indoor location and movement sensors may have potential, but are yet to be applied in this context [96].

Technology available within homes is changing rapidly and this also has implications for measurement of home media. For example electronic gaming may now be active or sedentary and portable media devices can be used in different places within the home [97]. However, in this review, only two observational studies included these more recent leisure technologies $[75,77]$.

\section{Future research directions}

Future studies on the influence of the home physical environment on children's sedentary behaviour and PA need to investigate home context specific outcomes. The investigation of objectively measured sedentary time at home is most important [98]. Sedentary time at home should also be categorised by the type of sedentary behaviour including screen based and other productive/ non-productive behaviours, such as reading and homework. This will assist to clarify whether children who spend less time in screen based behaviours are overall less sedentary at home, or whether they do more of other non-screen sedentary behaviours [33]. For PA, time spent in objectively measured PA levels at home and activity behaviours that occur specifically at home such as active play, AVG play and household chores are most relevant for future research.

Investigation of the home physical environment lacks objective assessment and is limited, except for equipment. Future research on the indoor and outdoor home environment should, where applicable, adopt approaches used to measure the outdoor built environment. For example, GIS can provide objective measures of house and 
yard size [99] and audits provide scope to collect more objective and detailed data inside the home [77]. Additionally, just as GPS has been applied to locate individuals in outdoor environments [95], newer measurement technologies, such as indoor location and movement sensors, provide an avenue to track the location of individuals in indoor space [96]. Future research should draw on established approaches from other fields and closely monitor the development of new technologies that have measurement potential within the home.

Both social and physical environmental factors influence children's sedentary behaviour and PA, yet how they interact within the home space remains unclear. Parents control many elements of the home physical environment. Future research should explore the relationships between parental behaviour, family rules, equipment and arrangement of the home space, to better understand how sedentary behaviour is influenced by the home environment and to inform the development of interventions.

Further intervention studies are highly recommended. We found only two types of interventions that changed the home physical environment. TV limiting devices reduced TV viewing, however, it is unknown which behaviours replace TV viewing and whether this strategy is successful in the longer term. Similarly, introducing an AVG showed promise, but any effects seemed to decay quickly. Interventions are required to determine the longer term effects of introducing TV limiting devices and AVGs on activity levels, and any unintended consequences on other physical activities and sedentary gaming. Other strategies such as changing the location of media equipment within the home, reconfiguring indoor spaces and substituting sedentary behaviours with active alternatives are lacking and should be explored. The key challenge for future research is to find acceptable active alternatives to traditionally sedentary behaviours within the home space.

\section{Strengths and limitations of the review}

This review included the best available evidence from both observational and intervention studies, identifying a larger number of studies with relevant home physical environmental variables than previous reviews. However, there are some limitations to the process and scope of this review. Firstly, some studies may have been missed due to the nature of the search terms and there may be some publication bias to studies with significant results. Secondly, independent and outcome measures were pooled into categories which were useful for summarising evidence, but did not differentiate between very specific environmental measures. For example, the home media equipment category included presence of a TV, presence of a computer, density of media equipment and number of TVs in the home. Thirdly, the summary of the home social environment in isolation should be interpreted with caution as it was limited to papers that also included home physical environmental factors and only the most common social home environmental factors were investigated. Also, individual factors were not included in the review. Finally, the pre-adolescent age group was identified as particularly relevant for investigation, although we acknowledge that this age group encompasses both children and adolescents as defined in previous reviews, and may limit comparability.

\section{Conclusion}

This review found that both physical and social environmental factors operating within the home space are important influences on children's sedentary behaviour and PA. Media equipment is associated with children's screen based sedentary behaviours. Changing the physical environment shows promise for reducing the sedentary nature of homes, although further interventions are needed to understand the broader impact of changes. Considering the substantial amount of time children spend at home, there has been little investigation of how the physical parameters of the home space may constrain or support children's sedentary behaviour and PA. Future studies should ideally include objective measures of the home and prioritise investigating environmental influences within the home space on objectively measured sedentary time at home and home context specific behaviours.

\section{Competing interests}

The authors declare that they have no competing interests.

\section{Authors' contributions}

CM contributed to the conceptualisation and design of the manuscript conducted the literature search, quality assessment of papers and analysis, and drafted the manuscript. GS, SF, RB, \& MR contributed to the conceptualisation of the manuscript and verification of papers, and provided critical feedback on the manuscript. All authors read and approved the final manuscript.

\section{Acknowledgements}

CM is supported by a Healthway Health Promotion Research Scholarship (\#21357). SF is supported by a Healthway Health Promotion Research Fellowship (\#21363). The authors would like to acknowledge the University of Western Australia for providing resources to enable the completion of the study.

\section{Author details}

${ }^{1}$ School of Sport Science, Exercise and Health, University of Western Australia, 35 Stirling Highway, Crawley, WA 6009, Australia. ${ }^{2}$ Applied Sports Technology Exercise Medicine Research Centre, School of Engineering, Swansea University, Singleton Park, Swansea SA2 8PP, Wales. ${ }^{3}$ Centre for Built Environment and Health, School of Population Health, University of Western Australia, 10 Stirling Highway, Crawley, WA 6009, Australia.

Received: 3 October 2012 Accepted: 14 August 2013

Published: 17 August 2013

\section{References}

1. Owen N, Healy GN, Matthews CE, Dunstan DW: Too much sitting: the population health science of sedentary behavior. Exerc Sport Sci Rev 2010, 38:105-113 
2. Carver A, Timperio A, Crawford D: Playing it safe: the influence of neighbourhood safety on children's physical activity - a review. Health Place 2008, 14:217-227.

3. Karsten L: It all used to be better? Different generations on continuity and change in urban children's daily use of space. Child Geogr 2005 3:275-290.

4. Hallal PC, Victora CG, Azevedo MR, Wells JCK: Adolescent physical activity and health. Sports Med 2006, 36:1019-1030.

5. Jiménez-Pavón D, Kelly J, Reilly JJ: Associations between objectively measured habitual physical activity and adiposity in children and adolescents: systematic review. Int J Pediatr Obes 2010, 5:3-18.

6. Andersen LB, Harro M, Sardinha LB, Froberg K, Ekelund U, Brage $S$, Anderssen SA: Physical activity and clustered cardiovascular risk in children: a cross-sectional study (The European Youth Heart Study). Lancet 2006, 368:299-304.

7. Marshall SJ, Biddle SJH, Gorely T, Cameron N, Murdey I: Relationships between media use, body fatness and physical activity in children and youth: a meta-analysis. Int J Obes 2004, 28:1238-1246.

8. Tremblay MS, LeBlanc AG, Kho ME, Saunders TJ, Larouche RC, Colley R, Goldfield G, Gorber SC: Systematic review of sedentary behaviour and health indicators in school-aged children and youth. Int J Behav Nutr Phys Act 2011, 8:98

9. Matthews CE, Chen KY, Freedson PS, Buchowski MS, Beech BM, Pate RR, Troiano RP: Amount of time spent in sedentary behaviors in the United States, 2003-2004. Am J Epidemiol 2008, 167:875-881.

10. Troiano RP, Berrigan D, Dodd KW, Masse LC, Tilert T, McDowell M: Physical activity in the United States measured by accelerometer. Med Sci Sports Exerc 2008, 40:181-188.

11. Tremblay MS, Colley RC, Saunders TJ, Healy GN, Owen N: Physiological and health implications of a sedentary lifestyle. Appl Physiol Nutr Metab 2010, 35:725-740.

12. Ridley K, Ainsworth BE, Olds TS: Development of a compendium of energy expenditures for youth. Int J Behav Nutr Phys Act 2008, 5:45.

13. Gorely T, Marshall SJ, Biddle SJH: Couch kids: correlates of television viewing among youth. Int J Behav Med 2004, 11:152-163.

14. van der Horst $K$, Chin A Paw MJM, Twisk JWR, van Mechelen W: A brief review on correlates of physical activity and sedentariness in youth. Med Sci Sports Exerc 2007, 39:1241-1250.

15. Sallis JF, Prochaska JJ, Taylor WC: A review of correlates of physical activity of children and adolescents. Med Sci Sports Exerc 2000, 32:963-975.

16. Pate RR, Mitchell JA, Byun W, Dowda M: Sedentary behaviour in youth. Br J Sports Med 2011, 45:906-913.

17. Stokols D: Establishing and maintaining healthy environments: toward a social ecology of health promotion. Am Psychol 1992, 47:6-22.

18. Sallis JF, Owen N, Fisher EB: Ecological Models of Health Behaviour. In Health Behaviour and Health Education: Theory, Research and Practice. 4th edition. Edited by Glanz K, Rimer BK, Viswanath K. San Francisco: Jossey-Bass; 2008:465-485

19. Giles-Corti B, Timperio AF, Bull FC, Pikora T: Understanding physical activity environmental correlates: increased specificity for ecological models. Exerc Sport Sci Rev 2005, 33:175-181.

20. Edwardson $\mathrm{CL}$, Gorely T: Parental influences on different types and intensities of physical activity in youth: a systematic review. Psycho/ Sport Exerc 2010, 11:522-535

21. Carver A, Timperio AF, Crawford DA: Neighborhood road environments and physical activity among youth: the CLAN study. J Urban Health 2008, 85:532-544.

22. Ding D, Sallis JF, Kerr J, Lee S, Rosenberg DE: Neighborhood environment and physical activity among youth - a review. Am J Prev Med 2011 41:442-455.

23. Veitch J, Bagley S, Ball K, Salmon J: Where do children usually play? A qualitative study of parents' perceptions of influences on children's active free-play. Health Place 2006, 12:383-393.

24. Hesketh K, Waters E, Green J, Salmon L, Williams J: Healthy eating, activity and obesity prevention: a qualitative study of parent and child perceptions in Australia. Health Promotion Int 2005, 20:19-26.

25. Sebire SJ, Jago R, Gorely T, Hoyos Cillero I, Biddle SJH: "If there wasn't the technology then I would probably be out everyday": a qualitative study of children's strategies to reduce their screen viewing. Prev Med 2011, 53:303-308
26. Jago R, Thompson JL, Page AS, Brockman R, Cartwright K, Fox KR: Licence to be active: parental concerns and 10-11-year-old children's ability to be independently physically active. J Public Health 2009, 31:472-477.

27. Granich J, Rosenberg M, Knuiman M, Timperio AF: Understanding children's sedentary behaviour: a qualitative study of the family home environment. Health Educ Res 2010, 25:199-210.

28. Davison KK, Lawson CT: Do attributes in the physical environment influence children's physical activity? A review of the literature. Int Behav Nutr Phys Act 2006, 3:19.

29. Ferreira I, van der Horst K, Wendel-Vos W, Kremers S, van Lenthe FJ, Brug J. Environmental correlates of physical activity in youth: a review and update. Obes Rev 2007, 8:129-154.

30. Australian Bureau of Statistics: Larger Dwellings, Smaller Households. Canberra: Commonwealth of Australia; 2007.

31. Roberts-Hughes R: The Case for Space: The Size of England's New Homes. London: Royal Institute of British Architects; 2011.

32. Jones AP, Coombes EG, Griffin SJ, van Sluijs EMF: Environmental supportiveness for physical activity in English schoolchildren: a study using global positioning systems. Int J Behav Nutr Phys Act 2009, 6:42

33. Biddle SJH, Marshall SJ, Gorely T, Cameron N: Temporal and environmental patterns of sedentary and active behaviors during adolescents' leisure time. Int J Behav Med 2009, 16:278-286.

34. Sener IN, Copperman RB, Pendyala RM, Bhat CR: An analysis of children's leisure activity engagement: examining the day of week, location, physical activity level, and fixity dimensions. Transportation 2008, 35:673-696.

35. Thornburg HD: Is early adolescence really a stage of development? Theory Into Pract 1983, 22:79-84.

36. Nader PR, Bradley RH, Houts RM, McRitchie SL, O'Brien M: Moderate-tovigorous physical activity from ages 9 to 15 years. JAMA 2008, 300:295-305.

37. Brodersen NH, Steptoe A, Boniface DR, Wardle J: Trends in physical activity and sedentary behaviour in adolescence: ethnic and socioeconomic differences. Br J Sports Med 2007, 41:140-144.

38. Rideout VJ, Foehr UG, Roberts DF: Generation M2: Media in the Lives of 8- to 18-Year-Olds. Menlo Park, California: Henry J. Kaiser Family Foundation; 2010.

39. Aeffect Inc: Review of Literature to Support Development of the Youth Media Campaign: Exploring How to Motivate Behavior Change Among Tweens in America. Lake Forest, Illinois: Department of Health and Human Services' Centers for Disease Control and Prevention; 2000.

40. National Institute for Health and Clinical Excellence: Methods for the Development of NICE Public Health Guidance (second edition). London: National Health Service; 2009.

41. Cui Z, Hardy LL, Dibley MJ, Bauman A: Temporal trends and recent correlates in sedentary behaviours in Chinese children. Int I Behav Nutr Phys Act 2011, 8:93.

42. Devis-Devis J, Peiro -Velert C, Beltran-Carrillo VJ, Tomas JM: Screen media time usage of 12-16 year-old Spanish school adolescents: effects of personal and socioeconomic factors, season and type of day. J Adolesc 2009, 32:213-231.

43. Granich J, Rosenberg M, Knuiman MW, Timperio A: Individual, social, and physical environment factors associated with electronic media use among children: sedentary behavior at home. J Phys Act Health 2011, 8:613-625.

44. Hardy LL, Baur LA, Garnett SP, Crawford D, Campbell KJ, Shrewsbury VA Cowell CT, Salmon J: Family and home correlates of television viewing in 12-13 year old adolescents: The Nepean Study. Int J Behav Nutr Phys Act 2006, 3:24

45. Hesketh K, Ball K, Crawford D, Campbell K, Salmon J: Mediators of the relationship between maternal education and children's TV viewing Am J Prev Med 2007, 33:41-47.

46. Hoyos Cillero I, Jago R: Sociodemographic and home environment predictors of screen viewing among Spanish school children. J Public Health 2011, 33:392-402.

47. Hume C, van der Horst K, Brug J, Salmon J, Oenema A: Understanding the correlates of adolescents' TV viewing: a social ecological approach. Int J Pediatr Obes 2010, 5:161-168.

48. Jago R, Page A, Froberg K, Sardinha LB, Klasson-Heggebo L, Andersen LB: Screen-viewing and the home TV environment: The European Youth Heart Study. Prev Med 2008, 47:525-529.

49. Norman GJ, Schmid BA, Sallis JF, Calfas KJ, Patrick K: Psychosocial and environmental correlates of adolescent sedentary behaviors. Pediatrics 2005, 116:908-916. 
50. Patriarca A, Di Giuseppe G, Albano L, Marinelli P, Angelillo IF: Use of television, videogames, and computer among children and adolescents in Italy. BMC Public Health 2009, 9:139.

51. Ramirez ER, Norman GJ, Rosenberg DE, Kerr J, Saelens BE, Durant N, Sallis JF: Adolescent screen time and rules to limit screen time in the home. J Adolesc Health 2011, 48:379-385.

52. Springer AE, Kelder SH, Barroso CS, Drenner KL, Shegog R, Ranjit N, Hoelscher DM: Parental influences on television watching among children living on the Texas-Mexico border. Prev Med 2010, 51:112-117.

53. van Sluijs EMF, Page A, Ommundsen Y, Griffin SJ: Behavioural and social correlates of sedentary time in young people. Br J Sports Med 2010, 44:747-755.

54. van Zutphen M, Bell AC, Kremer PJ, Swinburn BA: Association between the family environment and television viewing in Australian children. J Paediatr Child Health 2007, 43:458-463.

55. Zabinski MF, Norman GJ, Sallis JF, Calfas KJ, Patrick K: Patterns of sedentary behavior among adolescents. Health Psychol 2007, 26:113-120.

56. Te Velde SJ, van der Horst K, Oenema A, Timperio A, Crawford D, Brug J: Parental and home influences on adolescents' TV viewing: a mediation analysis. Int J Pediatr Obes 2011, 6:364-372.

57. Willoughby T: A short-term longitudinal study of internet and computer game use by adolescent boys and girls: prevalence, frequency of use, and psychosocial predictors. Dev Psychol 2008, 44:195-204.

58. Aarts MJ, Wendel-Vos W, van Oers HAM, van de Goor IAM, Schuit AJ: Environmental determinants of outdoor play in children - a large-scale cross-sectional study. Am J Prev Med 2010, 39:212-219.

59. Erwin HE, Woods AM, Woods MK, Castelli DM: Children's environmental access in relation to motor competence, physical activity, and fitness. J Teach Phys Educ 2007, 26:404-415.

60. Haerens L, Craeynest M, Deforche B, Maes L, Cardon G, De Bourdeaudhuij l: The contribution of home, neighbourhood and school environmental factors in explaining physical activity among adolescents. J Environ Public Health 2009, 2009:320372.

61. Kerr J, Norman GJ, Sallis JF, Patrick K: Exercise aids, neighborhood safety, and physical activity in adolescents and parents. Med Sci Sports Exerc 2008, 40:1244-1248.

62. Li M, Dibley MJ, Sibbritt D, Yan H: Factors associated with adolescents' physical inactivity in Xi'an City, China. Med Sci Sports Exerc 2006, 38:2075-2085.

63. Maddison R, Hoorn SV, Jiang Y, Ni Mhurchu C, Exeter D, Dorey E, Bullen C, Utter J, Schaaf D, Turley M: The environment and physical activity: the influence of psychosocial, perceived and built environmental factors. Int J Behav Nutr Phys Act 2009, 6:19.

64. McMinn AM, van Sluijs EMF, Nightingale CM, Griffin SJ, Cook DG, Owen CG, Rudnicka AR, Whincup PH: Family and home correlates of children's physical activity in a multi-ethnic population: the cross-sectional Child Heart and Health Study in England (CHASE). Int J Behav Nutr Phys Act 2011, 8:11

65. Page AS, Cooper AR, Griew P, Jago R: Independent mobility, perceptions of the built environment and children's participation in play, active travel and structured exercise and sport: The PEACH Project. Int J Behav Nutr Phys Act 2010, 7:17.

66. Ridgers ND, Graves LEF, Foweather L, Stratton G: Examining influences on boy's and girls' physical activity patterns: The A-CLASS Project. Pediatr Exerc Sci 2010, 22:638-650

67. Spinks A, Macpherson A, Bain C, McClure R: Determinants of sufficient daily activity in Australian primary school children. J Paediatr Child Health 2006, 42:674-679.

68. Trang NHHD, Hong TK, Dibley MJ, Sibbritt DW: Factors associated with physical inactivity in adolescents in Ho Chi Minh City, Vietnam. Med Sci Sports Exerc 2009, 41:1374-1383.

69. Veitch J, Salmon J, Ball K: Individual, social and physical environmental correlates of children's active free-play: a cross-sectional study. Int J Behav Nutr Phys Act 2010, 7:11.

70. Wong BYM, Cerin E, Ho SY, Mak KK, Lo WS, Lam TH: Adolescents' physical activity: competition between perceived neighborhood sport facilities and home media resources. Int J Pediatr Obes 2010, 5:169-176.

71. Crawford D, Cleland V, Timperio A, Salmon J, Andrianopoulos N, Roberts R, Giles-Corti B, Baur L, Ball K: The longitudinal influence of home and neighbourhood environments on children's body mass index and physical activity over 5 years: The CLAN study. Int J Obes 2010, 34:1177-1187.
72. Wilson DK, Lawman HG, Segal M, Chappell S: Neighborhood and parental supports for physical activity in minority adolescents. Am J Prev Med 2011, 41:399-406.

73. Hume C, Salmon J, Ball K: Children's perceptions of their home and neighborhood environments, and their association with objectively measured physical activity: a qualitative and quantitative study. Health Educ Res 2005, 20:1-13.

74. Roemmich JN, Epstein LH, Raja S, Yin L: The neighborhood and home environments: disparate relationships with physical activity and sedentary behaviors in youth. Ann Behav Med 2007, 33:29-38.

75. Rosenberg DE, Sallis JF, Kerr J, Maher J, Norman GJ, Durant N, Harris SK, Saelens BE: Brief scales to assess physical activity and sedentary equipment in the home. Int J Behav Nutr Phys Act 2010, 7:10.

76. Salmon J, Timperio A, Telford A, Carver A, Crawford D: Association of family environment with children's television viewing and with low level of physical activity. Obes Res 2005, 13:1939-1951.

77. Sirard JR, Laska MN, Patnode CD, Farbakhsh K, Lytle LA: Adolescent physical activity and screen time: associations with the physical home environment. Int J Behav Nutr Phys Act 2010, 7:82.

78. Delmas C, Platat C, Schweitzer B, Wagner A, Oujaa M, Simon C: Association between television in bedroom and adiposity throughout adolescence. Obesity 2007, 15:2495-2503.

79. French SA, Gerlach AF, Mitchell NR, Hannan PJ, Welsh EM: Household obesity prevention: Take Action - a group-randomized trial. Obesity 2011, 19:2082-2088

80. Robinson TN, Borzekowski DL: Effects of the SMART classroom curriculum to reduce child and family screen time. J Commun 2006, 56:1-26.

81. Todd MK, Reis-Bergan MJ, Sidman CL, Flohr JA, Jameson-Walker K, Spicer-Bartolau T, Wildeman K: Effect of a family-based intervention on electronic media use and body composition among boys aged 8-11 years: a pilot study. J Child Health Care 2008, 12:344-358.

82. Madsen KA, Yen S, Wlasiuk L, Newman TB, Lustig R: Feasibility of a dance videogame to promote weight loss among overweight children and adolescents. Arch Pediatr Adolesc Med 2007, 161:105-107.

83. Owens SG, Garner JC 3rd, Loftin JM, van Blerk N, Ermin K: Changes in physical activity and fitness after 3 months of home Wii Fit use. J Strength Cond Res 2011, 25:3191-3197.

84. Graves LEF, Ridgers ND, Atkinson G, Stratton G: The effect of active video gaming on children's physical activity, behavior preferences and body composition. Pediatr Exerc Sci 2010, 22:535-546.

85. Ni Mhurchu CN, Roberts V, Maddison R, Dorey E, Jiang Y, Jull A, Tin ST: Effect of electronic time monitors on children's television watching: pilot trial of a home-based intervention. Prev Med 2009, 49:413-417.

86. Maddison R, Foley L, Ni Mhurchu C, Jiang YN, Jull A, Prapavessis H, Hohepa M, Rodgers A: Effects of active video games on body composition: a randomized controlled trial. Am J Clin Nutr 2011, 94:156-163.

87. Ni Mhurchu C, Maddison R, Jiang $Y$, Jull A, Prapavessis $H$, Rodgers A: Couch potatoes to jumping beans: a pilot study of the effect of active video games on physical activity in children. Int J Behav Nutr Phys Act 2008, 5:8.

88. Chin A Paw MJM, Jacobs WM, Vaessen EPG, Titze S, van Mechelen W: The motivation of children to play an active video game. J Sci Med Sport 2008, 11:163-166.

89. Goldfield GS, Mallory R, Parker T, Cunningham T, Legg C, Lumb A Parker K, Prud'homme D, Gaboury I, Adamo KB: Effects of open-loop feedback on physical activity and television viewing in overweight and obese children: a randomized, controlled trial. Pediatrics 2006, 118:157-166.

90. Dunton GF, Kawabata K, Intille S, Wolch J, Pentz MA: Assessing the social and physical contexts of children's leisure-time physical activity: an ecological momentary assessment study. Am J Health Promot 2012, 26:135-142.

91. Rainham DG, Bates CJ, Blanchard CM, Dummer TJ, Kirk SF, Shearer CL: Spatial classification of youth physical activity patterns. Am J Prev Med 2012, 42:87-96.

92. Biddle SJH, Atkin AJ, Cavill N, Foster C: Correlates of physical activity in youth: a review of quantitative systematic reviews. Int Rev Sport Exerc Psychol 2011, 4:25-49.

93. Owen N, Sugiyama T, Eakin EE, Gardiner PA, Tremblay MS, Sallis JF: Adults' sedentary behavior: determinants and interventions. Am J Prev Med 2011, 41:189-196.

94. Jago R, Baranowski T, Baranowski JC: Observed, GIS, and self-reported environmental features and adolescent physical activity. Am J Health Promot 2006, 20:422-428. 
95. Krenn PJ, Titze S, Oja P, Jones A, Ogilvie D: Use of global positioning systems to study physical activity and the environment - a systematic review. Am J Prev Med 2011, 41:508-515.

96. Zhu C, Sheng W: Motion- and location-based online human daily activity recognition. Pervasive Mob Comput 2011, 7:256-269.

97. Jago R, Sebire S, Gorely T, Hoyos Cillero I, Biddle SJH: "I'm on it 24/7 at the moment": a qualitative examination of multi-screen viewing behaviours among UK 10-11 year olds. Int I Behav Nutr Phys Act 2011, 8:85.

98. Tandon PS, Zhou C, Sallis JF, Cain KL, Frank LD, Saelens BE: Home environment relationships with children's physical activity, sedentary time, and screen time by socioeconomic status. Int I Behav Nutr Phys Act 2012, 9:88.

99. Hall T: Goodbye to the backyard? The minimisation of private open space in the Australian outer-suburban estate. Urban Pol Res 2010, 28:411-433

doi:10.1186/1479-5868-10-99

Cite this article as: Maitland et al:: A place for play? The influence of the home physical environment on children's physical activity and

sedentary behaviour. International Journal of Behavioral Nutrition and Physical Activity 2013 10:99.

\section{Submit your next manuscript to BioMed Central and take full advantage of:}

- Convenient online submission

- Thorough peer review

- No space constraints or color figure charges

- Immediate publication on acceptance

- Inclusion in PubMed, CAS, Scopus and Google Scholar

- Research which is freely available for redistribution 